\title{
Kardiovaskuläre Folgeereignisse
}

\section{Zulassungserweiterung für Statin plus Ezetimib}

Aktuelle Daten der IMPROVE-ITStudie mit 18.144 Patienten nach stationärer Behandlung wegen eines akuten Koronarsyndroms [Murphy SA et al. JACC. 2016;67:353-61] bestätigen erneut, dass die Gabe von Ezetimib zusätzlich zu Simvastatin im Vergleich zur Statin-Monotherapie die Zahl kardiovaskulärer Ereignisse reduziert. Die neue Auswertung schloss nicht nur Primärereignisse, sondern auch Folgeereignisse und Gesamtereignisse ein.

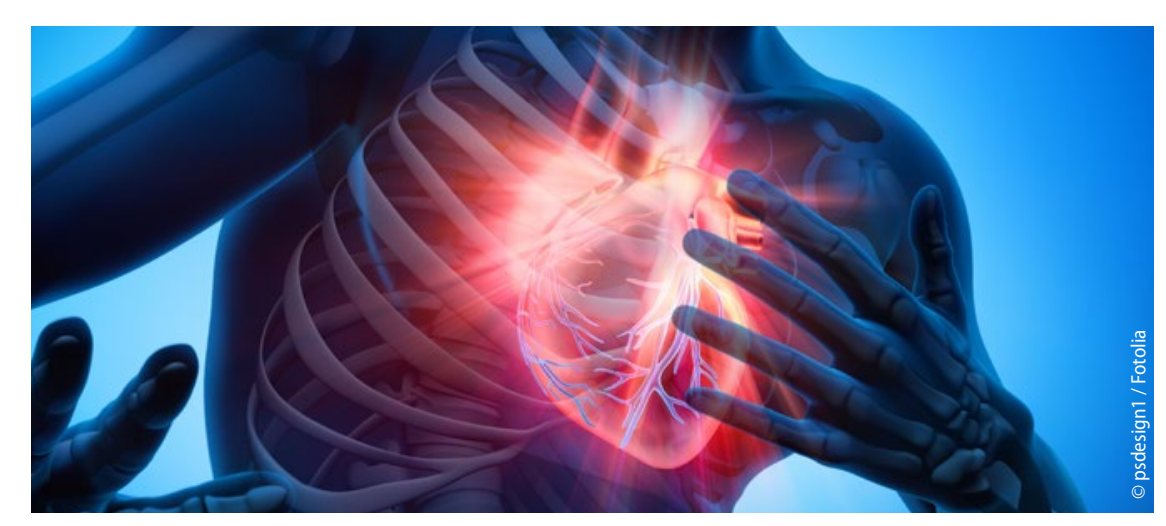

Die Gesamtzahl nicht-tödlicher Myokardinfarkte ging um 13\% zurück.

Dies entspricht oft dem klinischen Verlauf von Hypercholesterinämie-Patienten mit sehr hohem kardiovaskulärem Risiko, da sie häufig mehr als ein Ereignis erleiden. Im medianen Follow-up von sechs Jahren zeigte sich, dass die Kombination Ezetimib plus Simvastatin die Zahl der Folgeereignisse im Vergleich zu einer Simvastatin-Monotherapie um 12\% reduzierte. Absolut gab es 1.990 Folgeereignisse in der Ezetimib/ Simvastatin-Gruppe und 2.241 in der
Placebo/Simvastatin-Gruppe. Die Risikoreduktion lag fast doppelt so hoch wie bei den Erstereignissen, für die sie bei 6,4\% lag [Cannon CP et al. N Engl J Med. 2015;372:2387-97].

\section{LDL-Senkung weiterführen}

Die neuen Daten verdeutlichen, wie wichtig es ist, nach einem kardiovaskulären Ereignis eine intensive LDL-Senkung fortzuführen, um auch Folgeereignisse verhindern zu können.

Diese positiven Ergebnisse haben nun Eingang in die Zulassung der zur Verfügung stehenden Kombinationspräparate gefunden: Atorvastatin plus Ezetimib (z.B. Tioblis ${ }^{\circledR}$ ) und Simvastatin plus Ezetimib (z.B. Goltor ${ }^{\circledR}$ ) sind jetzt auch angezeigt zur Risikoreduktion kardiovaskulärer Ereignisse bei Patienten mit koronarer Herzkrankheit (KHK) und akutem Koronarsyndrom in der Vorgeschichte, und zwar unabhängig von einer Statin-Vorbehandlung.

Red.

\section{Exazerbationsschutz}

\section{Steroide könnten bei COPD bald weitgehend ausgedient haben}

_ Die Hinweise mehren sich, dass inhalative Kortikosteroide (ICS) bei der chronisch-obstruktiven Lungenerkrankung (COPD) weitgehend ausgedient haben. Potente Bronchodilatatoren scheinen akute Exazerbationen mindestens ebenso wirksam verhindern zu können - bei besserem Nutzen-Risiko-Profil.

In der großen SUMMIT-Studie konnte 2015 die Fixkombination aus ICS und lang wirksamen $\beta 2$-Agonisten (LABA) die Überlebensprognose nicht verbessern. „Für ICS spricht nur die Senkung der Exazerbationsrate - aber können andere Wirkstoffe das nicht auch?", fragte Prof. Carl-Peter Criée, Göttingen.

Gute Daten liegen für die duale Bronchodilatation mit einem LABA und einem lang wirksamem Anticholinergikum (LAMA) vor - bisher allerding nicht für Patienten mit hohem Exazerbationsrisiko. Im Mai nun werden die Ergebnisse der FLAME-Studie veröffentlicht, um diese Lücke zu schließen.

\section{Vorteil für LAMA/LABA-Kombi}

Für die Studie wurden 3.362 Patienten mit moderater bis schwerer COPD und Exazerbationsanamnese ein Jahr lang mit der LAMA/LABA-Fixkombination Indacaterol/Glycopyrronium (Ultibro ${ }^{\oplus}$ Breezhaler $^{\circledast}$ ) oder mit der ICS/LABAKombi Fluticason/Salmeterol behandelt. $\mathrm{Zu}$ hören ist, dass LAMA/LABA beim primären Endpunkt, der Exazerbationsrate, besser abgeschnitten hat.
„Wir werden die ICS bei COPD neu bewerten müssen“, meinte Criée. Gänzlich obsolet würden sie wohl nicht, Vielmehr müsse man Patienten identifizieren, die wirklich von ihnen profitieren. Eosinophile im Blut könnten dafür ein Marker sein - auch beim Asthma könne man bei Eosinophilie ja ein gutes Ansprechen auf ICS erwarten.

Exazerbationen allein heranzuziehen, um die ICS-Indikation zu begründen, scheint jedenfalls nicht gerechtfertigt Zumal ICS für COPD-Patienten Risiken bergen. Criée erinnerte an die deutlich erhöhte Pneumonierate, die in Studien immer wieder gefunden wurde.

\section{Manuela Arand}

- 5. Novartis Pneumologentage; Berlin, 29.-31. Januar 2016 